\title{
COMBINATORIAL EXPRESSION FOR UNIVERSAL VASSILIEV LINK INVARIANT
}

\section{SERGEY PIUNIKHIN}

\author{
Harvard University
}

February 18,1993

\begin{abstract}
The most general R-matrix type state sum model for link invariants is constructed. It contains in itself all R-matrix invariants and is a generating function for "universal" Vassiliev link invariants. This expression is more simple than Kontsevich's expression for the same quantity, because it is defined combinatorially and does not contain any integrals, except for an expression for "the universal Drinfeld's associator".
\end{abstract}

\section{INTRODUCTION}

Vassiliev knot invariants were invented [Va1], [Va2] in attempt to construct some natural basis in the space $H^{0}$ (Imbeddings: $S^{1} \rightarrow \mathbf{R}^{3}$ ) of all knot invariants, using natural stratification of discriminant set of nonimbeddings: $S^{1} \rightarrow \mathbf{R}^{3}$ and a kind of infinite-dimensional Alexander duality. Although the question whether Vassiliev knot invariants can distinguish any two knots is still opened, this language is the most appropriate in studying classical knot and link invariants. All known knot and link invariants: Alexander polynomial [Ro], [Co], Jones polynomial [Jo1], Kauffman polynomial [Ka1], [Ka2], HOMFLY polynomial [HOMFLY] and all their generalizations [Tu1];[Tu2];[Re1];[RT] and Milnor's $\mu$-invariants [Mi1], [Mi2], which generalize Gauss linking number -can be incorporated into this scheme (see [BL], [Li1], [Li2], [BN5]).

The space of Vassiliev knot invariants of fixed order $\mathrm{n}$ (factored through the space of invariants of order $n-1$ ) can be described pure combinatorially as a space of functions on the set of assignments of n pairs of points on $S^{1}$ subject to certain linear relations. These relations were first written in [BL], the fact, that this set of relations is complete was proved by Kontsevich [Ko1], using explicit integral presentation of "universal" Vassiliev invariant $I_{n}$ of order n, which takes values in a certain linear space $F_{n}$ generated by "Vassiliev diagrams". This space has also very nice description in terms of Feynman diagrams of perturbative Chern-Simons theory [BN1], [BN2], [BN3], [Ko1]; the graded linear space $F=$ $\oplus_{n} F_{n}$ admits Hopf algebra structure [Ko1], [BN3] (Kontsevich Hopf algebra). 
The space of primitive elements in this Hopf algebra is generated by connected Feynman diagrams [Pi2], [Ko2], [BN1].

The generating function $I=\sum_{n=0}^{\infty} h^{n} I_{n}$ of "universal order n" - Vassiliev invariants gives us the "universal" $\mathrm{F}$-valued Vassiliev invariant (here $\mathrm{h}$ is formal parameter), where $I_{n}(\mathrm{~K})$ is certain $\mathrm{n}$-fold integral over the knot $\mathrm{K}$ (Kontsevich integral [Ko1]). At the moment nobody is able to calculate explicitly $\mathrm{I}(\mathrm{K})$ for any non-trivial knot $\mathrm{K}$.

The aim of the present paper is to give more simple expression for this quantity, which can be calculated explicitly in all orders in h. , if one can calculate the universal Drinfeld's "associator" [Dr1],[Dr2]. This expression models state sum expression [Re1], [Tu1], [Jo2] for knot polynomials $P_{g, V}\left(q^{ \pm 1}\right)$ (here $q=e^{h}$ ) constructed from (semi)simple Lie algebra $g$ and its irreducible representation V, after forgetting about $\mathrm{g}$ and $\mathrm{V}$. Connection between $P_{g, V}\left(q^{ \pm 1}\right)$ and Vassiliev knot invariants was found in [Li1] : If $P_{g, V}(\mathrm{~h})=\sum_{n=0}^{\text {infty }} P_{g, V, n} h^{n}$ than $P_{g, V, n}$ is Vassiliev invariant of order n. Explicit state sum expression for $P_{g, V, n} \in F_{n}$ was deduced in [Pi1], using results of [BN1], where the same quantity was derived from perturbative Chern-Simons theory [BN3], [AS], [GMM], [Ko3]. The generating function $P_{g, V}(\mathrm{~h})=\sum_{n=0}^{\text {infty }} P_{g, V, n} h^{n}$ can be explicitly calculated as a state sum expression in terms of knot diagram [Re1], [Tu1], [Jo2].

The question is, whether it is possible to forget about Lie algebra $\mathrm{g}$ and representation V and to write the "universal" state sum expression $P=\sum_{n=0}^{i n f t y} h^{n} P_{n}$ with values in Kontsevich Hopf algebra $\mathrm{F}$ and then, in order to obtain $P_{g, V}$, apply Bar-Natan rules to $P=\sum_{n=0}^{\text {infty }} h^{n} P_{n} ; P \in F$ to obtain C-valued invariant $P_{g, V, n}$. Half of this program (forgetting about $\mathrm{V}$ but not about g) was accomplished by Reshetikhin [Re2] where he constructed knot invariant $P_{g} \in Z(U(g))$ belonging to the center of quantum universal enveloping algebra $\mathrm{U}(\mathrm{g})$. Here $P_{g, V}=\operatorname{Tr}_{V}(P)$. This construction also works for an arbitrary quasitriangular Hopf algebra [Dr3] instead of $\mathrm{U}(\mathrm{g})$ [Re2] and for arbitrary quasitriangular quasiHopf algebra [Dr1]. The next stage is to forget about this quasi-Hopf algebra. There are two ways to do this. The first one is to forget about $\mathrm{U}(\mathrm{g})$ "in the integral expression for monodromy of Knizhnik-Zamolodchikov equation [KZ], [K1], [TK]. This is the way how Kontsevich obtained his famous integral expression for I [Ko1]. The second way is to use "local" braid group representation $B_{m} \rightarrow(U(g) \otimes C[h])$ using Drinfeld's R-matrix $\left.R=e^{\frac{h t}{2}} \in(U(g)) \otimes C[h]\right)^{\otimes 2}$ and "associator" $\Phi \in(U(g)) \otimes C[h])^{\otimes 3}[\mathrm{Dr} 1],[\mathrm{Dr} 2]$ and then to forget about $\mathrm{U}(\mathrm{g})$. This way will give us the desired expression.

The parer is organized as follows:

In section 2 some preliminary definitions are given and "the universal prounipotent" braid group representation is constructed.

In section $3 \mathrm{~F}$-valued "Markov trace" in this representation is constructed and the fact that it is a generating function for "universal" Vassiliev link invariants is proved. Multiplicative property of this "universal" invariant with respect to connected sums is proved. Generalization for string link invariants 
is also given.

In section 4 some discussions are made.

\section{PRELIMINARIES}

Following D.Bar-Natan, we shall call BN-diagram several directed circles (called Wilson loops) and certain finite number of dashed lines (called gauge propagators). The propagators and Wilson lines are allowed to meet in two types of vertices: one type (called $R^{2} g$-vertices) in which a propagator ends on one of the Wilson loops; and another (called $g^{3}$-vertices) connecting three propagators. We assume, that one of two possible orders of gauge propagators meeting in any $g^{3}$-vertex is specified. Each BN-diagram can be uniquely presented by isotopy type of plane diagrams such as in fig.1.

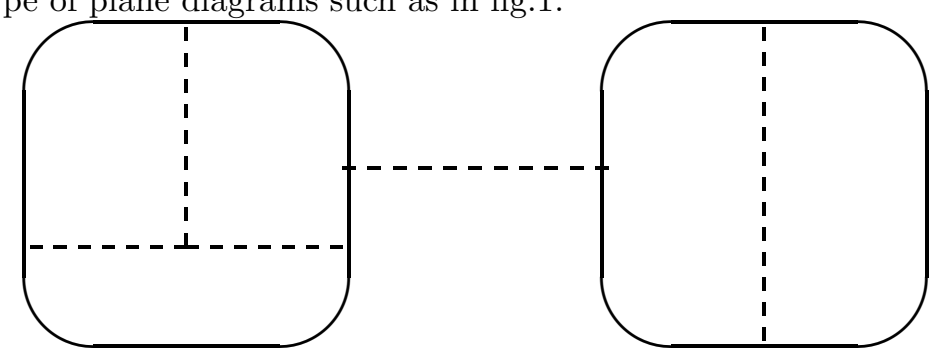

fig.1

Here, as usual, we assume, that all four-valent vertices are fake, and the counterclockwise order in each $g^{3}$-vertex is fixed. For instance, the orders of propagators on graphs in fig. $2 \mathrm{a}$ and fig. $2 \mathrm{~b}$ are different.

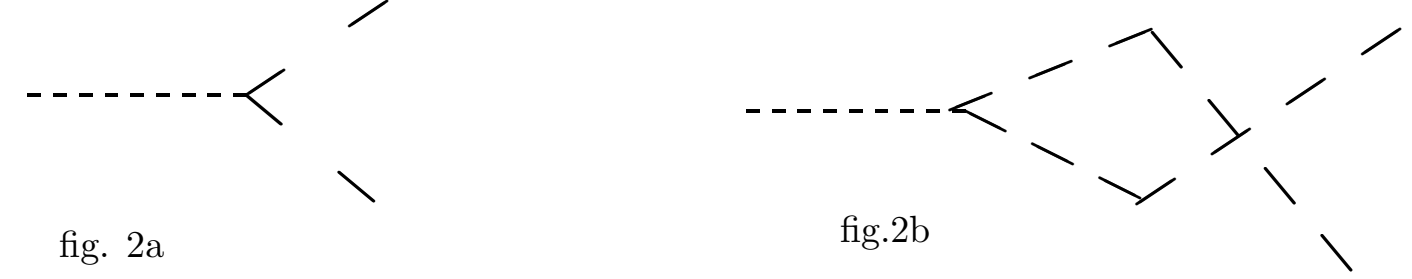

Let $\mathrm{K}$ be some ring $\mathbf{Z}_{1} K_{1} \mathbf{C}$.

\section{Definition.}

A function $\mathrm{C}:(B N-$ diagrams $) \rightarrow K$ is called weight system if two axioms are satisfied:

$$
C(I)=C(H)-C(X)
$$

where I, H, X, are BN-diagrams, identical everywhere except some small ball, where they look as in fig.3 


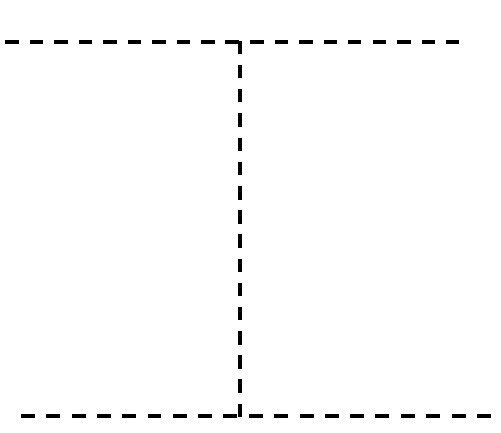

I

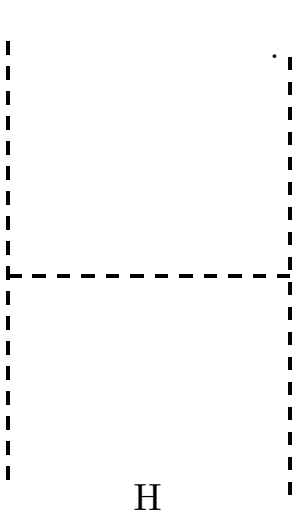

fig.3

$$
C(S)=C(T)-C(U)
$$

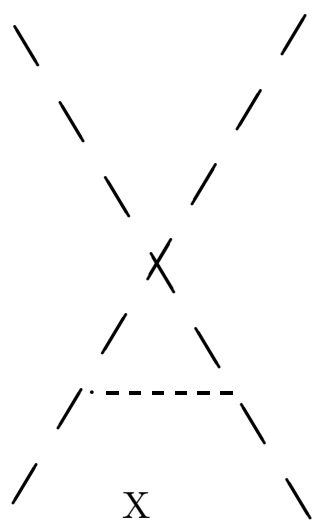

where S, T, U are BN-diagrams, identical everywhere except some small ball, where they look as in fig.4
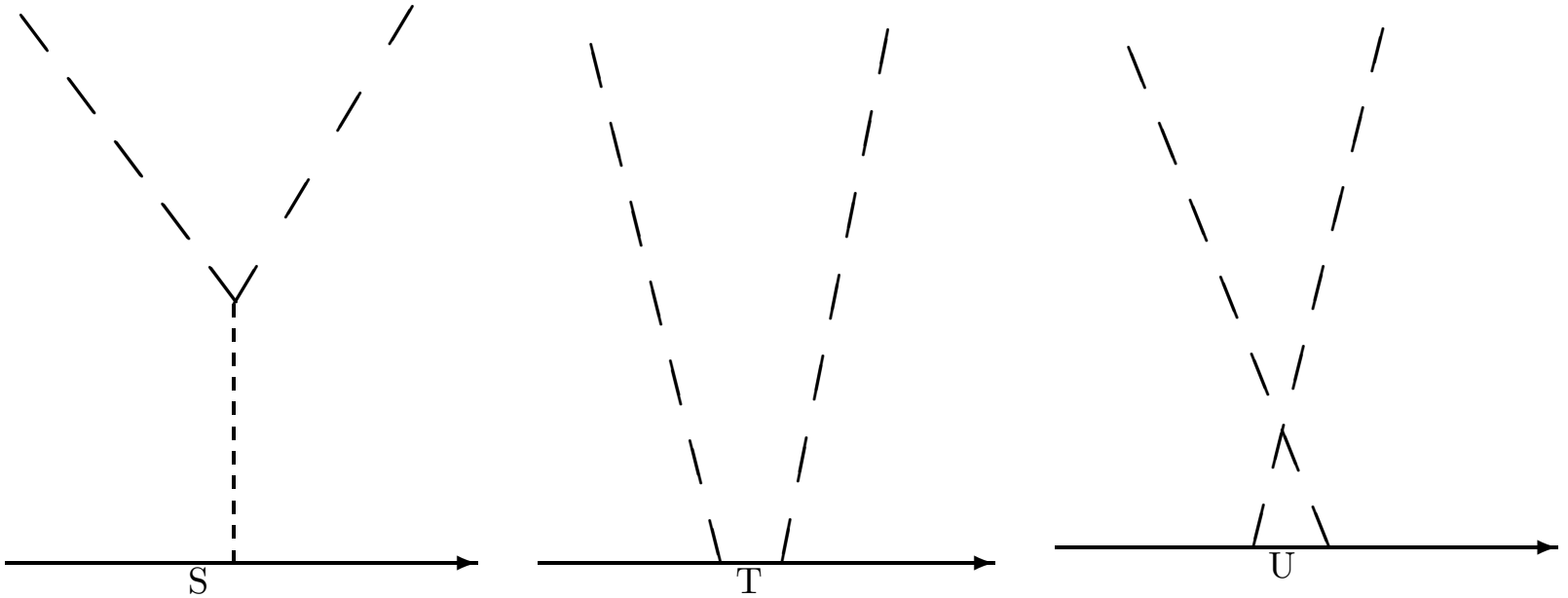

fig. 4

\section{Definition.}

Following Vassiliev [Va1], [Va2] and Birman-Lin [BL] we shall call BNdiagram with $2 \mathrm{n} R^{2} g$-vertices and without $g^{3}$-vertices Vassiliev [n]-diagram and BN-diagram with 2n-2 $R^{2} g$-vertices and with one $g^{3}$-vertex Vassiliev < $n>$-diagram . Let $\mathrm{D}$ be $\left\langle n>\right.$-diagram. Let us define [n]-diagrams $D_{1+}$ 

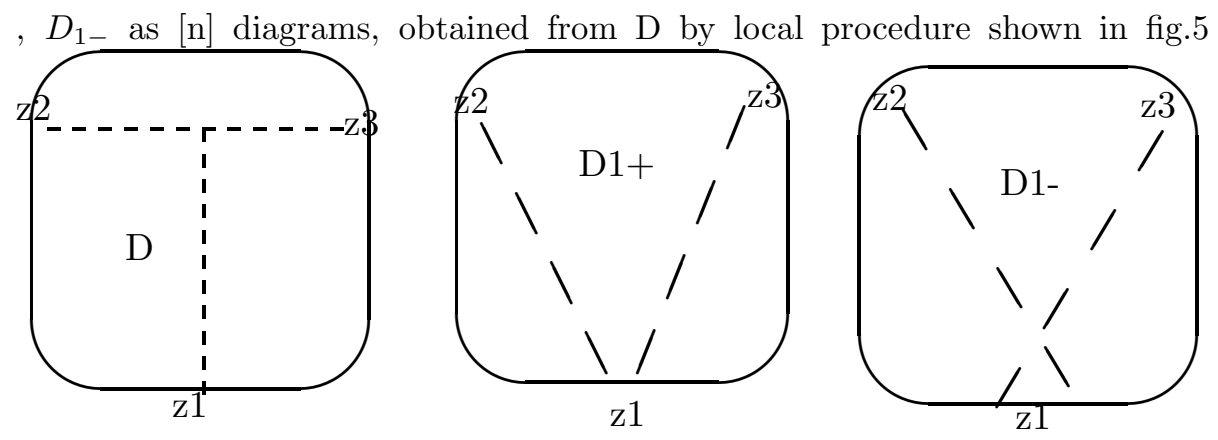

fig.5

(The [n]-diagrams $D_{2+}, D_{2-}, D_{3+}, D_{3-}$ are defined by changing $z_{1}$ on $z_{2}$ and $z_{3}$ respectively).

Definition. [BL], [Va1], [Va2], [Ko1]. Let $D^{s}(s \in \mathbf{N})$ be free K-module, generated by the set of s-Wilson-loop-Vassiliev [n]-diagrams; let $F^{s}$ be its factormodule, generated by relations

$$
D_{1+}-D_{1-}=D_{2+}-D_{2-}
$$

(D runs over $<n>$-diagrams). Let us denote $F_{0}^{s}=K ; F^{s}=\oplus_{n} F_{n}^{s}$, and let us identify $1 \in K=F_{0}^{s}$ with (the unique) s-Wilson-loop Vassiliev [0]-diagram.

Theorem 2.1. [Ko1], [Ar], [BN1] K-module $F_{n}^{s}$ is isomorphic to factor of free module $D_{n}^{s}$ generated by $(\mathrm{n}+\mathrm{s})$-loop $\mathrm{BN}$-diagrams by relations

$$
I=H-X
$$

where I, $H, X$, are BN-diagrams, identical everywhere except some small ball, where they look as in fig. 3 and

$$
S=T-U
$$

where $S, T, U$ are closed diagrams, identical everywhere except some small ball, where they look as in fig.4 .

In fact $F^{1}$ can be provided by the structure of graded Hopf algebra [Ko1] and we shall call it Kontsevich Hopf algebra. If it will not lead to confusion, we'll omit the superscript 1 and write $F=F_{1}$.

Kontsevich Hopf algebra $F$ acts on $F^{s}$ (taking connected sum along Wilson loop) in s different ways commuting with each other [BN5], thus we have graded action of $F^{\otimes s}$ on $F^{s}$.

Let $A=\oplus_{n} A_{n}$ be factor-algebra of Kontsevich algebra by the ideal, generated $F_{1}$ (this K-module is has rank one and is generated by the single Vassiliev [1]-diagram. Let us denote this diagram by $t \in F_{1}$ ). Since the element $\mathrm{t}$ is primitive, A is also Hopf algebra. It well-known [Ko1] that the space $A_{n}^{*}$ dual 
to $A_{n}$ is canonically isomorphic to the space $V_{n}$ of Vassiliev framed knot invariants of order $\mathrm{n}$ factored by the space $V_{n-1}$. The map $V_{n} / V_{n-1} \rightarrow A_{n}^{*}$ is evaluation of knot invariant on singular embeddings with $\mathrm{n}$ double points [Va1], [Va2], [BL] which gives linear function $V_{n} / V_{n-1} \otimes A_{n} \rightarrow \mathbf{C}$. The inverse map $I_{n}: A_{n}^{*} \rightarrow V_{n} \rightarrow V_{n} / V_{n-1}$ was first constructed in [Ko1] and is called "Kontsevich integral". The aim of this paper is to construct (formally another) inverse map $J_{n}: A_{n}^{*} \rightarrow V_{n}$ which does not contain any integrals and which has simple combinatorial expression.

Definition. Let $X^{m}(m \in \mathbf{N})$ be graded completion of Lie algebra $\oplus_{n} X_{n}^{m}$, with generators $t^{i j}(i<j)$ of degree 1 and relations

$$
\left[t^{i j} ; t^{i k}+t^{j k}\right]=0
$$

This algebra is dual to Sulivan minimal model of "configuration space" $\left(\mathbf{C}^{\mathbf{m}}\right.$ without diagonals) and its universal enveloping algebra $U X^{m}$ is prounipotent completion of the group algrbra or pure braid group (see [K2] and references therein). Kohno [K1] used this algebra in order to write the most general form of Knizhnik-Zamolodchikov equation [KZ]

$$
\frac{d \Psi}{d z_{i}}=\hbar \sum_{j \neq i} \frac{t^{i j}}{z_{i}-z_{j}} \Psi
$$

where $\psi$ is $U X^{m}$-valued meromorphic function on $\left(\mathbf{C}^{\mathbf{m}}\right.$ without diagonals), $\hbar=\frac{h}{2 \pi i}$ Relations (2.6) are imposed in order to preserve zero-curvature condition

$$
\left[\frac{d}{d z_{i}}-\hbar \sum_{j \neq i} \frac{t^{i j}}{z_{i}-z_{j}} ; \frac{d}{d z_{k}}-\hbar \sum_{l \neq k} \frac{t^{k l}}{z_{k}-z_{l}}\right]=0
$$

which allows us to construct monodromy representation of pure braid group in the group $\exp \left(X^{m}\right)_{1} U X^{m}$. This representation is nonlocal and its matrix elements are certain multiple integrals . Following Drinfel'd [D1] let us put $m=3$ and write differential equation

$$
\frac{d G(x)}{d x}=\hbar\left(\frac{t^{12}}{x}+\frac{t^{23}}{x-1}\right) G(x) \in U X^{3}
$$

Than any solution $\Psi\left(z_{1}, z_{2}, z_{3}\right)$ of KZ-equation (2.7) with $\mathrm{m}=3$ has the form

$$
\left(z_{1}-z_{3}\right)^{\hbar\left(t^{12}+t^{13}+t^{23}\right)} G \frac{\left(z_{1}-z_{2}\right)}{\left(z_{1}-z_{3}\right)}
$$

where $\mathrm{G}$ satisfies (2.9) Let $G_{1}$ and $G_{2}$ be solutions of (2.9) defined when $0<$ $x<1$ with the asymptotic behavior

$$
G_{1}(x) \approx x^{\hbar t^{12}}(x \rightarrow 0)
$$


and

$$
G_{2}(x) \approx(x-1)^{\hbar t^{23}}(x \rightarrow 1)
$$

. Then

$$
G_{1}=G_{2} \Phi_{k z}
$$

for some $\Phi_{k z} \in \exp \left(X^{3}\right)_{1} U X^{3}$ and

$$
W_{1}=W_{2} \Phi_{k z}
$$

where $W_{1}$ and $W_{2}$ are solutions of (2.7) with $\mathrm{m}=3$ defined in the domain $z_{1}>$ $z_{2}>z_{3}$ with the asymptotic behavior

$$
W_{1} \approx\left(z_{1}-z_{2}\right)^{\hbar t^{12}}\left(z_{1}-z_{3}\right)^{\hbar\left(t^{13}+t^{23}\right.}
$$

for $\left(z_{1}-z_{2}\right)<<\left(z_{1}-z_{3}\right)$ and

$$
W_{2} \approx\left(z_{2}-z_{3}\right)^{\hbar t^{23}}\left(z_{1}-z_{3}\right)^{\hbar\left(t^{13}+t^{12}\right.}
$$

for $\left(z_{2}-z_{3}\right)<<\left(z_{1}-z_{3}\right)$.

As it was proved in [Dr1], [Dr2]

$$
\Phi_{k z}=\phi_{k z}\left(\hbar t^{12}, \hbar t^{23}\right)
$$

, where $\phi_{k z}(A, B)$ is some element of graded completion of tensor algebra with two generators $\mathrm{A}$ and $\mathrm{B} ; \log \left(\phi_{k z}(A, B)\right)=P_{k z}(A, B)$ belongs to the graded completion of free Lie algebra withtwo generators A and B; and

$$
P_{k z}(B, A)=-P_{k z}(A, B)
$$

Algebra $U X^{m}$ can be imbedded in the algebra $A_{k z}^{m}$ of Feynman diagrams (see [BN1],[BN5]) of the form depicted on fig 6 .

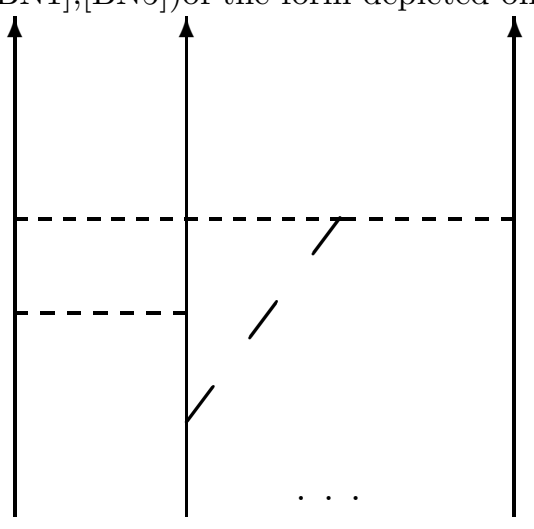

m upward pointed arrows

fig.6 
These diagrams are defined in the same way as usual BN-diagrams, but they have $\mathrm{m}$ upward pointed Wilson lines instead of one Wilson loop. Here $t^{i j}$ is presented by BN-diagram on fig.7.

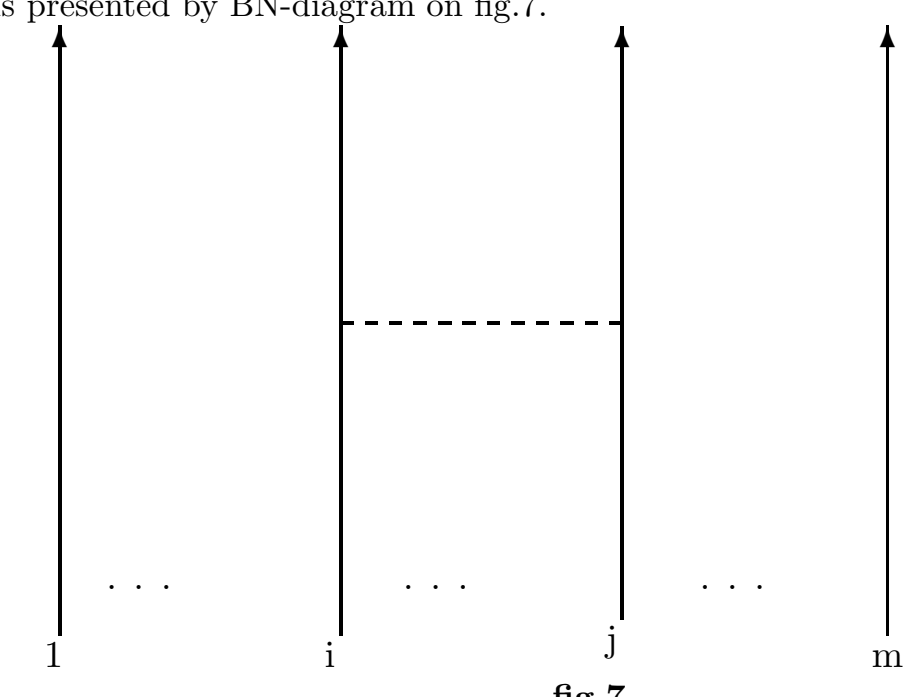

and the diagram with $2 \mathrm{n}$ vertices (this number is always even) is said to be if degree $n$. The multiplication in this algebra of diagrams is just putting one diagram over another. It is easy to see that above defined grading and multiplication in $A_{k z}^{m}$ are compatible with those of $U X^{m}$.

We'll need for ous purposes to define a semi-direct product $Y^{m}$ of the group algebra $\mathbf{C S}_{\mathbf{m}}$ of symmetric group and $A_{k z}^{m}$ as follows: $\mathrm{Y}$ is generated as a linear space by pairs (x, s); x is diagram from $A_{k z}^{m}, s \in S_{m}$ with multiplication

$$
\left(x_{1}, s_{1}\right)\left(x_{2}, s_{2}\right)=\left(x_{1} s\left(x_{2}\right), s_{1} s_{2}\right)
$$

Here we suppose that symmetric group acts on $A_{k z}^{m}$ by permutations of strings. Algebra $Y^{m}$ has an important subgroup

$$
G^{m}=S_{m} * \exp \left(X^{m}\right)_{1} Y^{m}
$$

Let $s_{i}(1 \leq i \leq m-1)$ be the standard generators of the braid group $B_{m}$ (if it not lead to confusion, we'll denote the elementary transpositions $s_{i} \in S_{m}$ by the same symbols.

Let us define representation $\rho: B_{m} \rightarrow G^{m} Y^{m}$ as follows:

$$
\begin{gathered}
\rho\left(s_{m-1}\right)=\left(e^{\frac{h t^{m-1, m}}{2}} ; s_{m-1}\right) \\
\rho\left(s_{i}\right)=\phi_{k z}^{-1}\left(h t^{i, i+1} ; h \sum_{s=i+2}^{m} t^{i+1, s}\right)\left(e^{\frac{h t^{i, i+1}}{2}} ; s_{i}\right) \phi_{k z}\left(h t^{i, i+1} ; h \sum_{s=i+2}^{m} t^{i+1, s}\right)
\end{gathered}
$$


if $1 \leq i \leq m-1$

This construction of representation $\rho$ is due to Drinfeld [Dr2]. It may be called "the universal" braid group representation since the group $G^{m}$ can be interpreted as prounipitent completion of $B_{m}$ [Dr2], [K2].

Above constructed representation $\rho$ is generalization of braid group action on quasitensor category [Dr1],[Dr2],[Re1]. To construct representation of the braid group $B_{m}$ one has to choose some configuration of parentheses in (nonassociative) product of $m$ symbols $x_{1}, \ldots, x_{m}$. Then, in order to define action of the braid group generator $s_{i}$, we should:

a) change the configuration of papentheses in order to have ...( $\left.x_{i} x_{i+1}\right) \ldots$ inside one pair of parantheses (this gives us some transition operator $\Phi_{\text {trans }}$, constructed out of "associator" $\Phi_{k z}$ according to Drinfeld's rules [Dr1],[Dr2]),

b) apply the Drinfeld's R-matrix $\left(e^{\frac{h t^{i, i+1}}{2}} ; s_{i}\right)$, and

c) return back to our initial configuration of parantheses (this gives us inverse operator to the operator $\Phi_{\text {trans }}$.

Formulas (2.15) and (2.16) correspond to one particular choice of configuration of papentheses, namely, $\left(x_{1}\left(x_{2}\left(\ldots\left(x_{m-1} x_{m}\right)\right) \ldots\right)\right.$ but any other choice is possible as well and gives us equivalent representation with some transition operator $\Phi_{\text {trans }}$ as an intertwinier. (If it don't lead to confusion, we'll denote all "transition operators" corresponding to transitions between different configurations of parentheses, by the same symbol $\left.\Phi_{\text {trans }}\right)$.

\section{TAKING THE TRACE}

It is well-known (see, for instance, [Bi] that any oriented s-component link $\mathrm{L}$ can be presented as a closed braid. Two braids $b_{1} \in B_{m 1}$ and $b_{2} \in B_{m 2}$ give under closure the same link iff they can be obtained from each other by finite sequence of Markov moves of two types:

$$
\begin{gathered}
b_{1} b_{2} \approx b_{2} b_{1} \in B_{m} \\
b \in B_{m} \approx b s_{m}^{ \pm 1} \in B_{m+1}
\end{gathered}
$$

Thus, any function $f: \bigcup_{m} B_{m} \rightarrow F^{s}$ for some commutative ring $\mathrm{K}$ and some Kmodule $F^{s}$ gives rise to some link invariant iff $\mathrm{f}$ takes the same values on braids, equivalent with respect to (3.1) and (3.2). Any framed link also can be presented as a closed braid. The analogues of Markov moves for braids which give under closure the same framed link (with blackboard framing [Tu], [Pi3], [Pi4] ) can also be described explicitly (see [Re2 ]). Here we give sufficient conditions for function $f: \bigcup_{m} B_{m} \rightarrow F^{s}$ to descend to some framed link invariant:

$$
\begin{gathered}
f\left(b_{1} b_{2}\right)=f\left(b_{2} b_{1}\right),\left(b_{1} ; b_{2} \in B_{m}\right) \\
f\left(b s_{m}^{ \pm 1}\right)=q_{i}^{ \pm 1} f(b) ; b \in B_{m}
\end{gathered}
$$


for some invertible element $q_{i} \in K$ (here $\mathrm{i}$ is number of the link component ). In the case of knots, let us put $F=\oplus_{n} F_{n}$ to be (graded) completion of Kontsevich Hopf algebra, considered as a module over graded completion of $\mathbf{C}\left[\mathbf{F}_{\mathbf{1}}\right]$. Here $q=e^{\frac{h t}{2}}$, where $\mathrm{t}$ is the standard generator in $F_{1}$ (see fig.8)

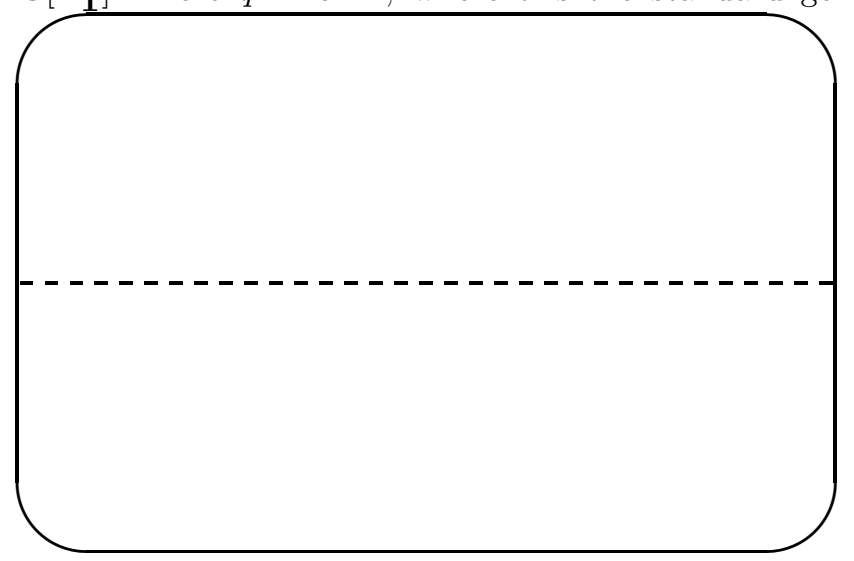

fig. 8

If we want to consider invariants of s-component links, we should take $F^{s}$ to be the $\mathbf{C}$-module $F^{s}$, generated by BN-diagrams with s Wilson loops . Here we put $q_{i}=e^{\frac{h t_{i}}{2}}$. Let us fix configuration of papentheses in (nonassociative) product of $2 \mathrm{~m}$ symbols $x_{1}, \ldots, x_{m}, y_{m}, \ldots, y_{1}$ as follows :

$$
\left(\left(x_{1}\left(\left(x_{2}\left(\ldots\left(\left(x_{m-1}\left(x_{m} y_{m}\right)\right) y_{m-1}\right) \ldots\right)\right) y_{2}\right)\right) y_{1}\right)
$$

Then, using this configuration of parantheses, let us define, in the spirit of formulas (2.15),(2.16) and remarks after them, representation $\widehat{\rho}: B_{m} \rightarrow G^{2 m_{1}} Y^{2 m}$ as restriction on $B_{m} B_{2 m}$ of representation $\Phi_{\text {trans }}^{-1} \rho \Phi_{\text {trans }}: B_{2 m} \rightarrow G^{2 m} Y^{2 m}$, where $\Phi_{\text {trans }}$ is transition operator between "the standard" configuration of parentheses on the set of $2 \mathrm{n}$ elements and the configuration (3.3).

Now let us suppose that the first $m$ Wilson lines in $Y^{2 m}$ are oriented "up" and the second $\mathrm{m}$ wilson lines are oriented "down". Then for any $m_{1} \mathbf{N}$ let us define map

$\tau: Y^{2 m} \rightarrow \bigcup_{s=1}^{m} F^{s}$ of graded linear spaces, which is defined simply by closure of $2 \mathrm{~m}$ directed Wilson lines in one Wilson loop according to the rule, given by underlying permutation $s \in \mathbf{S}_{\mathbf{m}}$ (see fig.9 as an example) 


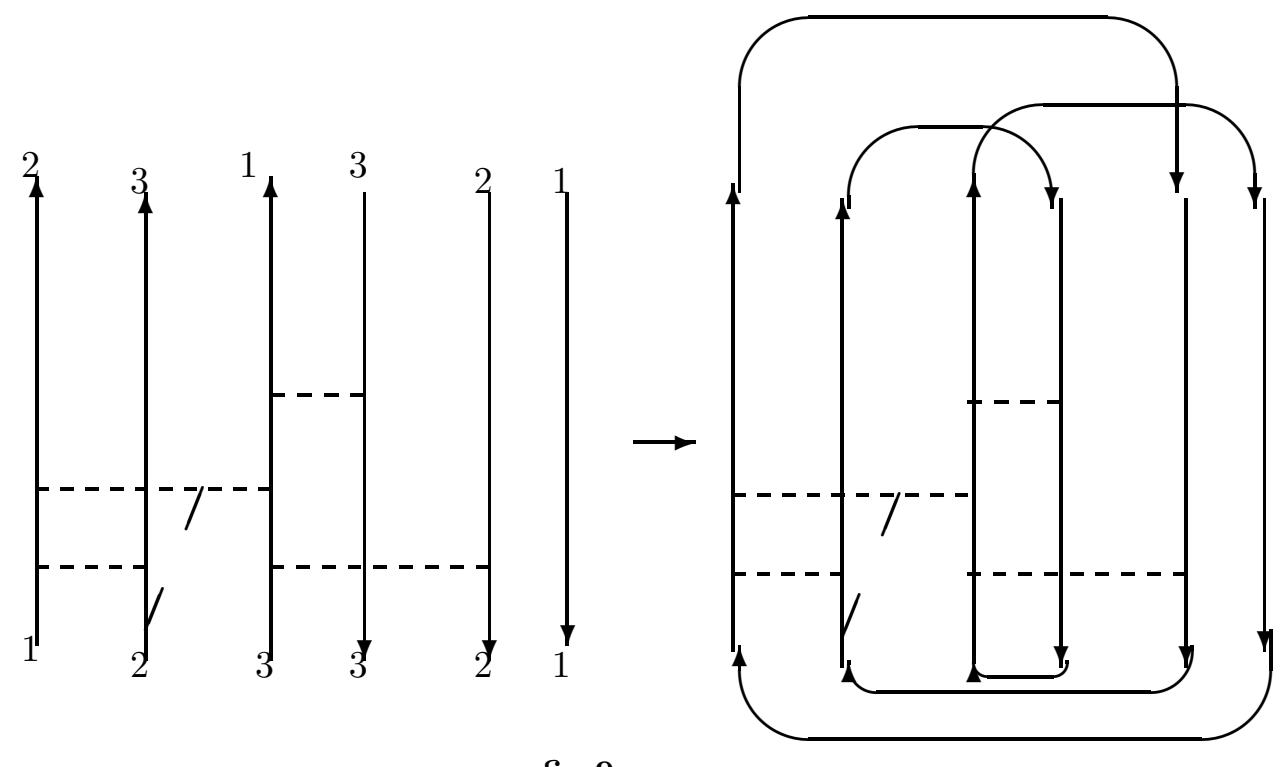

fig.9

if it will not lead to confusion, we'll not distingoish braids in $B_{m}$ and their images in $Y^{2 m}$. We will also suppose number of components s to pe fixed, omit index $\mathrm{m}$ and write $\tau: \bigcup_{m} B_{m} \rightarrow F^{s}$

Let $b_{1} \in B_{m_{1}} ; b_{2} \in B_{m_{2}}$ be two braids, $b_{2}$ gives knot under closure, and let $\left(b_{1} * b_{2}\right) \in B_{m_{1}+m_{2}-1}$ be the braid, obtained from $b_{1}$ and $b_{2}$ by the procedure shown on fig.10.
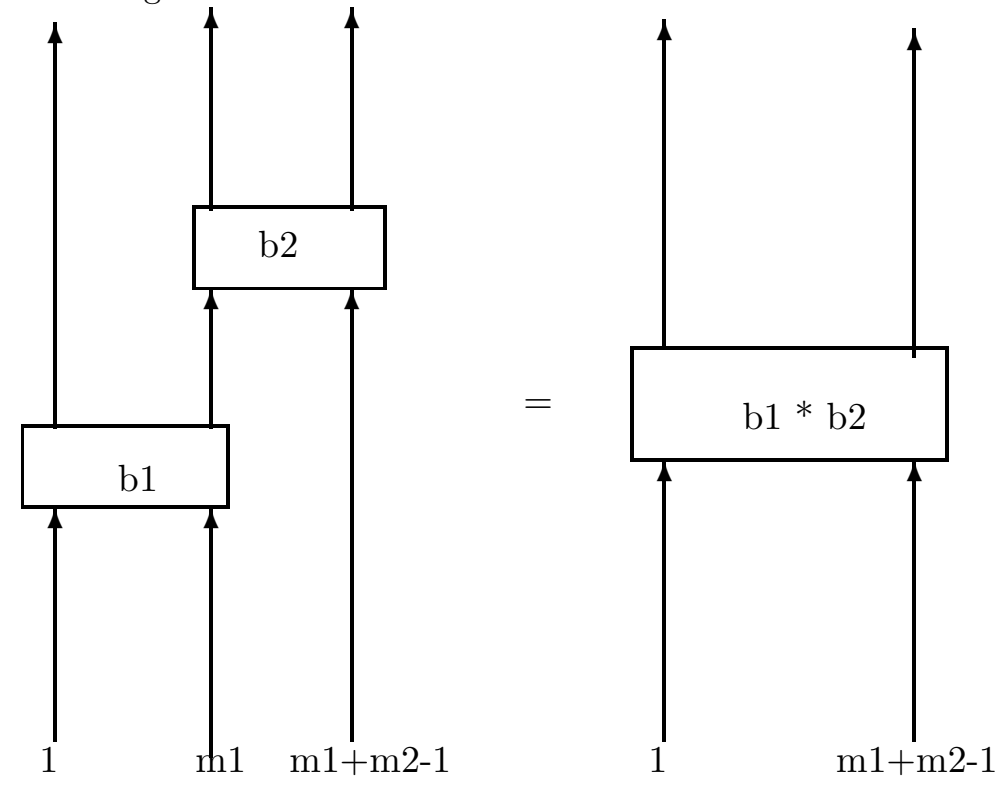
fig.10

Theorem 3.1.

$$
\tau\left(b_{1} * b_{2}\right)=\tau\left(b_{2}\right) * \tau\left(b_{1}\right)
$$

, where ${ }^{*}$ be the action of $F$ on $F^{s}$ (on the s-th component).

Proof. geometrically obvious from (3.3),fig.9 and fig.10.

Let $q=e^{\frac{h t}{2}}$ and let $\mu \in F$ be the image of Drinfeld's associator $\Phi_{k z} \in$ $\exp \left(X^{3}\right)_{1} U X^{3}$ under "the closure map" shown on fig.11.

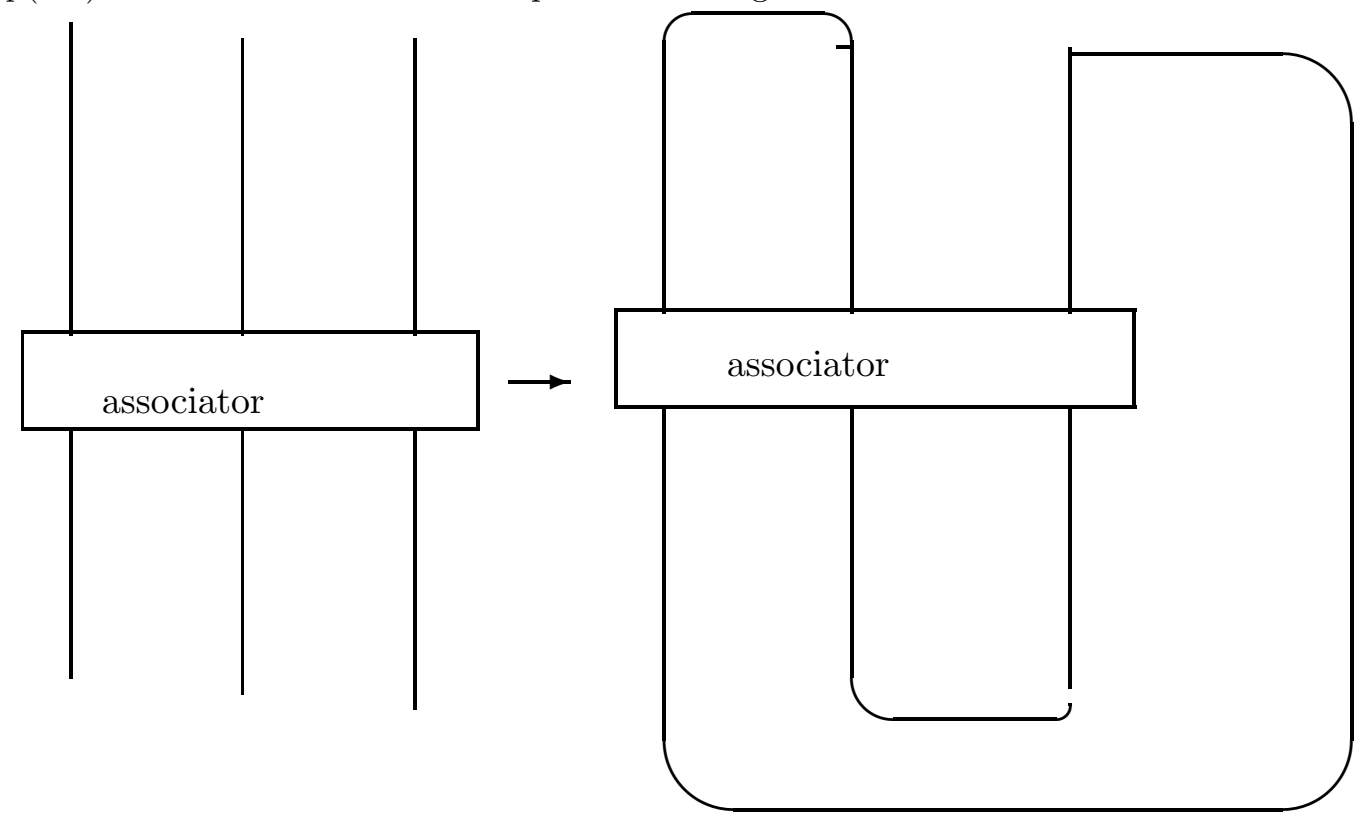

fig.11

Remark. Above defined $\mu$ is equal to the value of generating function of Kontsevich integrals on the Morse knot shown on fig.12.

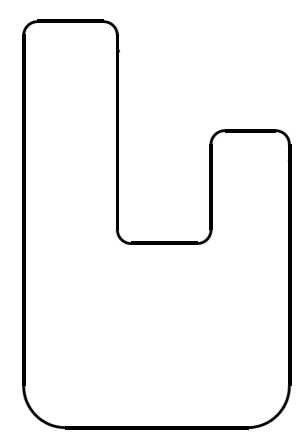

fig.12 
Lemma 3.2. Let $s_{1}$ be the standard generator of $B_{2}$. Then $\tau\left(s_{1}^{ \pm 1}\right)=q^{ \pm 1} \mu$

We give here pictorial proof:
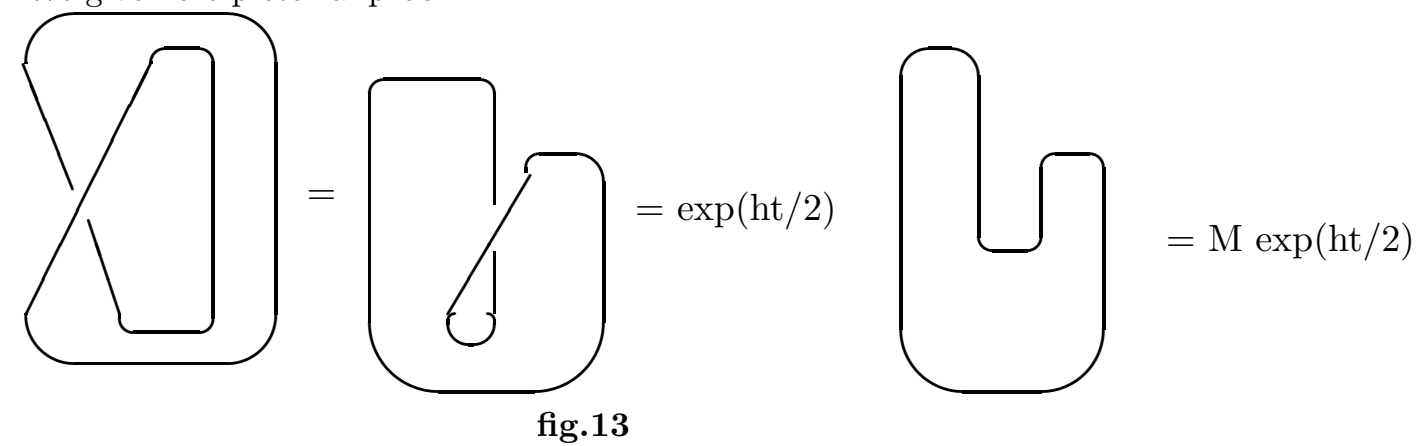

The first identity in fig.13 follows from identity on fig.14
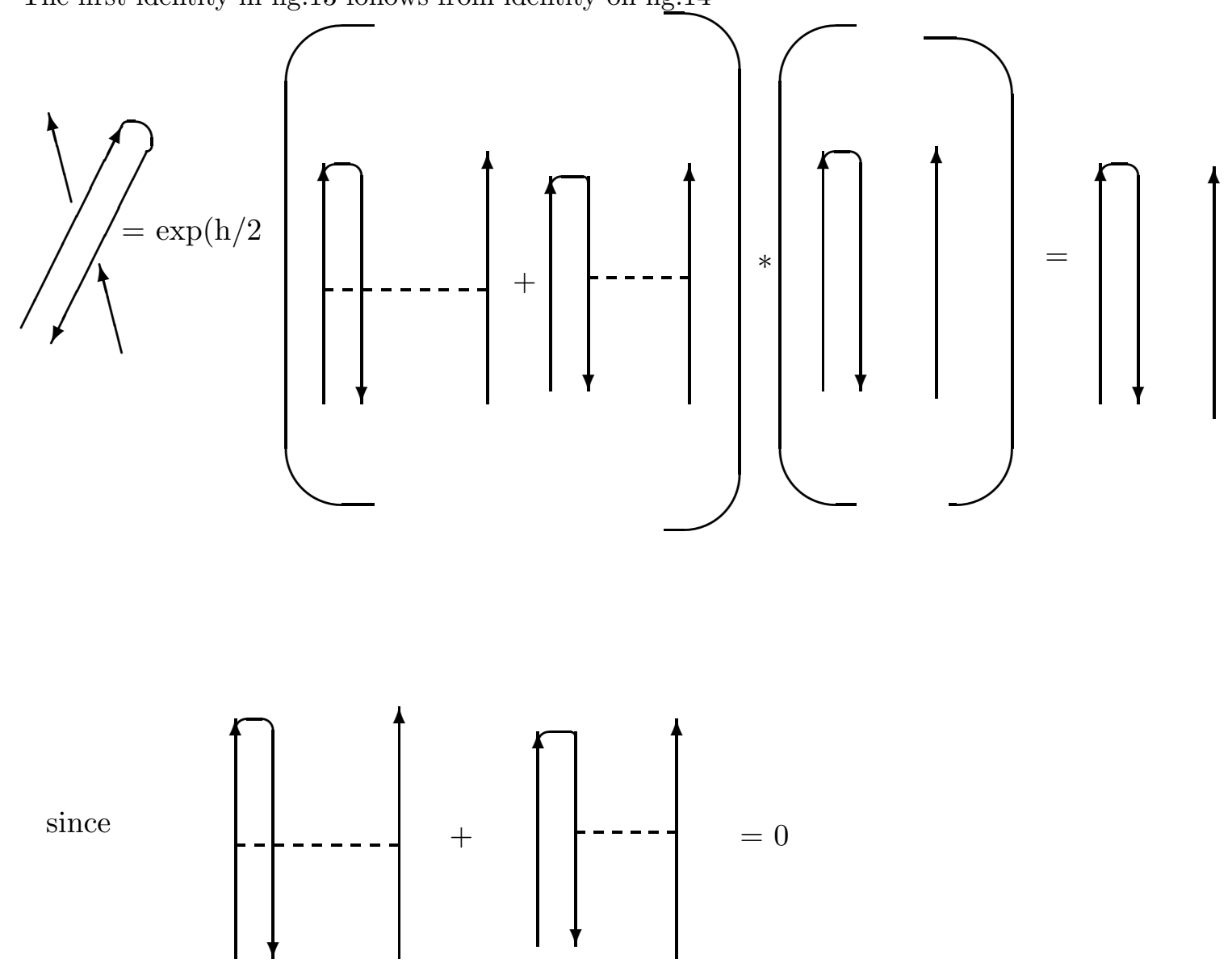

fig.14 
Let $\mathbf{J}: \mathbf{B}_{\mathbf{m}} \rightarrow \bigcup_{\mathbf{S}} \mathbf{F}^{\mathbf{S}}$ be equal to $(\mu)^{1-m} \tau: B_{m} \rightarrow \bigcup_{s} F^{s}$.

Lemma 3.3. The map $\mathbf{J}$ is "Markov trace" i.e., it satisfies (3.1A) and (3.2A).

Proof.

a) the property $(3.1 \mathrm{~A})$ is geometrically obvious.

b) the property (3.2A) follows from theorem 3.1 and lemma 3.2. from which (3.2A) with $q=e^{\frac{h t}{2}}$ can easily be seen. The lemma is proved.

Let $\mathbf{J}$ be above defined framed link invariant. Let us consider its perturbative expansion: $J=\sum_{n=0}^{\infty} h^{n} J_{n}$

Lemma 3.4. $J_{n}$ is $F_{n}^{s}$-valued Vassiliev framed link invariant of order n. Proof:

Since for any braid $b \in B_{m}$ if $\widehat{\rho}(b)=\sum_{n=0}^{\infty} x_{n}(b) h^{n} 1 Y^{2 m}$, that $x_{n}(b) 1 Y^{2 m}$ has degree $\mathrm{n}$ in $Y^{2 m}$ (this fact is true for the generators $s_{i} \in B_{m}$ and thus, for any $\left.b \in B_{m}\right)$, then for any framed oriented link $\mathbf{L} J_{n}(\mathbf{L})$ also has degree $\mathrm{n}$. Thus $J_{n}(\mathbf{L}) \in \mathbf{F}_{\mathbf{n}}^{\mathbf{S}} \mathbf{F}^{\mathbf{S}}$. Let $\mathbf{L}$ be singular imbedding of $\left(\mathbf{S}^{\mathbf{1}}\right)^{\mathbf{S}}$ to $\mathbf{R}^{\mathbf{3}}$ with $(\mathrm{n}+1)$ double crossing points. Than it can be presented as a closure of "generalized braid" [Pi1], [Ba] (braid where in some places generatots $s_{i}$ are changed on the generators $a_{i}$, depicted on fig.15.

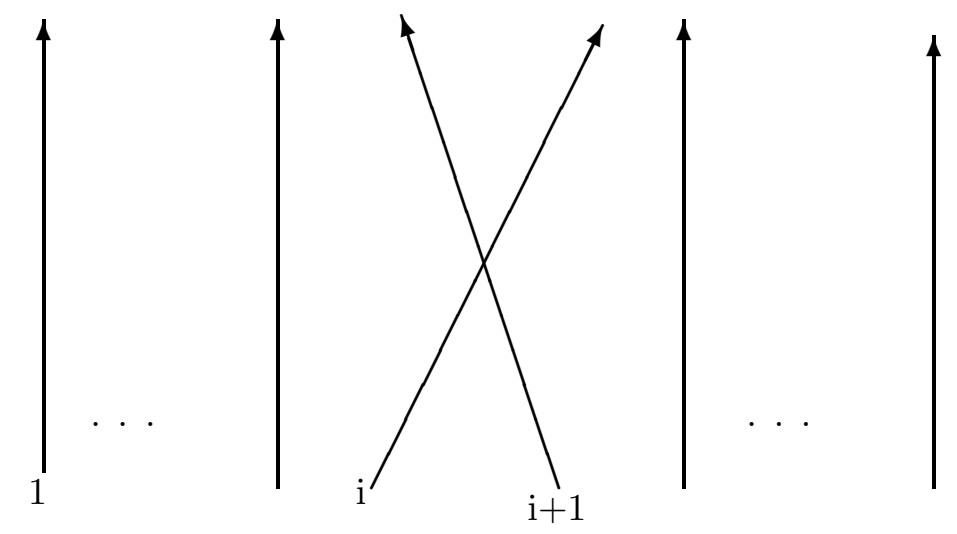

fig.15

The representation $\rho: B_{m} \rightarrow Y^{m}$ can be extended to these "generalized braids" by formula

$$
\rho\left(a_{i}\right)=\rho\left(s_{i}\right)-\rho\left(s_{i}^{-1}\right)
$$

(and the representation $\widehat{\rho}: B_{m} \rightarrow Y^{2 m}$ can be extended to "generalized braids" by the same formula).

(3.5), (2.14) and (2.15) imply that

$$
\rho\left(a_{m-1}\right)=2 \operatorname{sh} \frac{h t^{m-1, m}}{2} ; s_{m-1}
$$




$$
\rho\left(a_{i}\right)=\phi_{k z}^{-1}\left(h t^{i, i+1} ; h \sum_{s=i+2}^{m} t^{i+1, s}\right)\left(2 s h \frac{h t^{i, i+1}}{2} ; s_{i}\right) \phi_{k z}\left(h t^{i, i+1} ; h \sum_{s=i+2}^{m} t^{i+1, s}\right)
$$

if $i<m-1$

Thus $\widehat{\rho}\left(a_{i}\right)$ are divisible by $\mathrm{h}$ in $Y^{2 m} \otimes \mathbf{C}[\mathbf{h}]$, which implies, that for any "generalized braid" $b \in B_{m}$ with $(\mathrm{n}+1)$ double crossing points $\widehat{\rho}(b)$ is divisible by $h^{n+1}$. Thus $\mathbf{J}(\mathbf{L})$ is divisible by $h^{n+1}$, which means that $J_{n}(\mathbf{L})=0$ for any singular embedding $\mathbf{L}$ with $(\mathrm{n}+1)$ double crossing points, i.e., $J_{n}$ is Vassiliev invariant of order $\mathrm{n}$. Lemma is proved.

Let $V_{n}^{s}$ be the space of Vassiliev invariants of framed s-component links of order $\mathrm{n}$. Then there is a natural map $f_{n}: V_{n}^{s} \rightarrow V_{n}^{s} / V_{n-1}^{s} \rightarrow\left(F_{n}^{s}\right)^{*}$, defined as follows [Va1], [Va2], [BL]. Let $\mathrm{g}$ be some Vassiliev invariant of order $\mathrm{n}$ and $\mathrm{D}$ be Vassiliev [n]-diagram. Then

$$
\left(J_{n}(g) ; D\right)=(g ; L(D))
$$

where L(D) be some singular embedding $\left(\mathbf{S}^{\mathbf{1}}\right)^{\mathbf{S}} \rightarrow \mathbf{R}^{\mathbf{3}}$ with $\mathrm{n}$ double crossing points and the underlying configuration of $\mathrm{n}$ points on $\left(\mathbf{S}^{\mathbf{1}}\right)^{\mathbf{S}}$ given by diagram D.

Theorem 3.5. The map $\left(J_{n} ; \ldots\right):\left(F_{n}^{s}\right)^{*} \rightarrow V_{n}^{s}$ is left inverse to $f_{n}$, and differs from its right inverse on Vassiliev invariant of order n-1.

Proof. It is sufficient to prove that for any singular embedding L: $\left(\mathbf{S}^{\mathbf{1}}\right)^{\mathbf{S}} \rightarrow$ $\mathbf{R}^{\mathbf{3}}$ with precisely $\mathrm{n}$ double points

$$
J_{n}(L)=D(L)
$$

where $\mathrm{D}(\mathrm{L})$ is Vassiliev [n]-diagram with $\mathrm{n}$ propagators, joining those points on $\left(\mathbf{S}^{\mathbf{1}}\right)^{\mathbf{S}}$, which are identified under L. Let us present L as a closure of some "generalized braid" $b \in B_{m}$. Then $\widehat{\rho}(b)$ is product of some terms of the form

$$
\begin{gathered}
\left(e^{\frac{h t^{i, i+1}}{2}} ; s_{i}\right) \\
\Phi_{\text {trans }}^{ \pm 1} \\
\left(2 \operatorname{sh} \frac{h t^{i, i+1}}{2} ; s_{i}\right)
\end{gathered}
$$

There are precisely $\mathrm{n}$ terms of forms of form (3.12). Since terms (3.10) and (3.11) have the form

$$
1+h X
$$

for some $X \in Y^{2 m} ; \mu^{ \pm 1}$ also has the form (3.13) for some $X \in F$; and the terms (3.12) have the form

$$
h t^{i, i+1}+h^{2} X
$$


for some $X \in Y^{2 m}$, then the expression for the coefficient in $h^{n}$ in perturbative expansion of $\widehat{\rho}(b)$ has only one term, which gives $J_{n}(\mathrm{~L})=\mathrm{D}(\mathrm{L})$, as desired. The theorem is proved.

Kontsevich Hopf algebra $\mathrm{F}$ has (graded) factor algebra $A=F / F_{1} F$. Then $A_{n}^{*}$ is canonically identified with the space of Vassiliev unframed knot invariants of order $\mathrm{n}$ factored through the space of invariants of order n-1. In the basis of Vassiliev [n]-diagrams in $F_{n}$ the projector P: $F_{n} \rightarrow A_{n}$ can be described explicitly [Pi2].

$$
P(D)=\sum_{k=0}^{n}(-t)^{n} \sum_{I} D_{I}
$$

Where $\mathrm{t}$ is the generator of $F_{1}$; the second sum in (3.15) is taken over all [k]subdiagrams $D_{I}$ of $\mathrm{D}$. The quantity $P(J)=\sum_{n=0}^{\infty} h^{n} P\left(J_{n}\right):$ Knots $\rightarrow \mathrm{F}$ is the generating function for "universal" (order $\mathrm{n}$ )-Vassiliev knot invariants and has the same formal properties than the generating function $I=\sum_{n=0}^{\infty} h^{n} I_{n}$ of Kontsevich integrals [Ko1].

Theorem 3.6. Let $K_{1}$ and $K_{2}$ be two oriented framed knots; $K_{1} * K_{2}$ be their connected sum. Then $J\left(K_{1} * K_{2}\right)=J\left(K_{1}\right) J\left(K_{2}\right)$.

Proof. It follows immediately from theotem 3.1 and the definition of $\mathrm{J}$.

\section{DISCUSSIONS}

At the moment there are three different expressions for the universal Vassiliev knot invariant, (the quantity, which satisfies conditions of theorems 3.3 and 3.6). The first one is constructed from perturbative expansion of monodromy of KZ-equation (Kontsevich integrals [Ko1]), the second one is constructed from perturbative Chern-Simons theory [Ko1], [BN3] (These integrals are highly singular. Their convergence is rather difficult to prove). The third construction is presented here(see also [Ko5] where the similiar combinatorial construction was given, using knot diagram and a point on it). This construction is the best one for computations.

The "universal Vassiliev invariant" in the form presented here can be evaluated purely combinatorially for any particular link L , if we know explicit expression for an "associator" $\Phi_{k z}=\phi_{k z}\left(\hbar t^{12}, \hbar t^{23}\right) \in X^{3}$ as a formal noncommutative power series in $\hbar t^{12}$ and $\hbar t^{23}$. The powerful algebraic techniques to find this expression for $\Phi_{k z}$ was developed in [Dr2].An "iterated integral" expression for "assosiator" was proposed in [BN6], which proves immediately equivalence of our approach with Kontsevich's one (see also [LM] where coincidence of co-

efficients $P_{s l_{m}, V_{f u n d}, n}$ of one-variable reductions of HOMFLY polynomial and Kontsevich integrals $I_{n}$, evaluated on the weight system $C_{s l_{m}, V_{f u n d}, n} \in F_{n}^{*}$ is proved).

The analogous problem for "Kontsevich integrals" is much more complicated and involves calculations with hypergeomertic type integrals [TK].In our 
approach only one such integral (for each $n \in \mathbf{N}$ ) should be calculated. The calculations in perturbative Chern-Simons theory are even more complicated and are hardly to be accomplished by direct methods.

Above defined construction $\tau$ of the universal Vassiliev invariant of link which can be presented as a closure of braid has a straightforvard gneralization on arbitrarylink diagram, and even on string link diagram [BN5].Roughly speaking, $\tau$ is decomposition of the generating function of Kontsevich integrals (berore inserting the correction factor $\mu^{1-m}$ ) in the product of "elemrntary" factors corresponding to decomposition of link diagram on "elementary" pieces.

Drinfeld's construction of representation $\rho: B_{m} \rightarrow G^{m}$ involving $\Phi_{k z}=$ $\phi_{k z}\left(\hbar t^{12}, \hbar t^{23}\right)$ in its definition uses only the following properties of $\phi_{k z}$ :

$$
\begin{gathered}
\phi_{k z}\left(\hbar t^{12}, \hbar\left(t^{23}+t^{24}\right)\right) \phi_{k z}\left(\hbar\left(t^{12}+t^{13}\right), \hbar t^{34}\right)= \\
=\phi_{k z}\left(\hbar t^{23}, \hbar t^{34}\right) \phi_{k z}\left(\hbar\left(t^{12}+t^{13}\right), \hbar\left(t^{24}+t^{34}\right)\right) \phi_{k z}\left(\hbar t^{12}, \hbar t^{23}\right) \in \exp \left(X^{4}\right) \\
e^{\frac{h t^{12}+h t^{13}}{2}}=\Phi^{312} e^{\frac{h t^{13}}{2}}\left(\Phi^{132}\right)^{-1} e^{\frac{h t^{23}}{2}} \Phi \in \exp \left(X^{3}\right) \\
e^{\frac{h t^{13}+h t^{23}}{2}}=\left(\Phi^{231}\right)^{-1} e^{\frac{h t^{13}}{2}} \Phi^{213} e^{\frac{h t^{12}}{2}}(\Phi)^{-1} \in \exp \left(X^{3}\right) \\
\Phi \in X^{3}
\end{gathered}
$$

Here $\Phi^{s}\left(s \in S_{3}\right)$ is the image of $\Phi \in U X^{3}$ under automorphism $s: t^{i j} \rightarrow$ $t^{s(i) s(j)}$ Thus, if we find any other solution of (5.1)-(5.4) we can also construct "the universal braid group representation" $\rho_{\phi}$ and "the universal Vassiliev knot invariant" $J^{\phi}$. If we put $\phi$ to be formal noncommutative power series with rational coefficients, we obtain the "universal Q-valued Vassiliev invariant (the quantity, which satisfies conditions of theorems 3.3 and 3.6 and the condition, that for any oriented s-component link L

$$
J_{n}^{\phi}(L){ }_{1} F_{n}^{s} \otimes \mathbf{Q} \mathbf{1} \mathbf{F}_{\mathbf{n}}^{\mathbf{S}} \otimes \mathbf{C}
$$

(here we use the definition of $F_{n}$ as an abelian group i.e., over $\mathbf{Z}$ ).

To understand better all these formally different "universal formulas" and make calculations with them one needs some unification theorem, stating, that all these quantities (with different $\phi$ ) either coincide or are some functions of each other. We conjecture existence of such a theorem.

\section{Acknowledgements}

I benefited much from discussions with D.Bar-Natan, V.Drinfeld, D.Kazhdan and expecially with M.Kontsevich to whom I express my sincere gratitude.

\section{Literature}

[AS] S.Axelrod, I.Singer. Chern-Simons perturbation theory.

MIT preprint, October 1991.

[Ar] V.I.Arnold. Private communication. April 1992.

[Ba] J.C.Baez, Link invariants of finite type and perturbation theory.

Wellesley college preprint, July 1992. 
[Bi] J.S.Birman, Braids, links and mapping class groups.

Ann.of Math.Studies, no 82, Princeton Univ.Press. Princeton, NJ, 1974.

[BL] J.S.Birman and X.-S.Lin, Knot polynomials and Vassiliev's invariants. Preprint.1991.

[BN1] D.Bar-Natan, On Vassiliev knot invariants. Preprint. August 1992.

[BN2] D.Bar-Natan, Weights of Feynmann diagrams and Vassiliev

knot invariants. Preprint.February 1991.

[BN3] D.Bar-Natan, Perturbative aspects of the Chern-Simons topological quantum field theory.Ph.D.thesis, Princeton Univ., June 1991, Dep.of Mathematics.

[BN4] D.Bar-Natan, Perturbative Chern-Simons theory. Preprint. August 1990.

[BN5] D.Bar-Natan, Vassiliev homotopy string link invariants.

Preprint.December 1992.

[BN6] D.Bar-Natan, Private communication.

[Co] J.H.Conway, An enumeration of Knots and Links and some of their Alge-

braic Properties. in Computational Problems Abstract Algebra, p.329-358,

New York, Pergamon Press, 1969.

[Dr1] V.G.Drinfeld, Quasi-Hopf algebras, Leningrad Math J.,vol.1 (1990)p.1419-1457.

[Dr2] On quasitriangular quasi-Hopf algebras and a group, closely connected with Gal(Q/Q). Leningrad Math J.,vol.2 (1991)p.829-860.

[Dr3] V.G.Drinfeld, Quantum groups, Proc. Intern. Congr.Math. Berkeley, 1(1986), p.798-820.

[GMM] E.Guadagnini, M.Marellini and M.Mintchev, Perturbative aspects of the Chern-Simons field theory. Phys.Lett. B227(1989)p.111

[HOMFLY]P.Freyd, D.Yetter, J.Hoste, W.B.R.Lickorish, K.Milett, A.Ocneanu. A new polynomial invariant of knots and links.

Bull. Amer. Math., Soc.(1985), v.12, No2, p.239-246.

[Jo1] V.F.R.Jones, A polynomial invariant for links via von Neumann algebras.

Bull. AMS, v.12(1985), p.103-110.

[Jo2] V.F.R.Jones, On knot invariants related to some statistical mechanical models. Pacific J.Math. v.137 (1989)p.311-334.

[K1] T.Kohno. Monodromy representations of braid groups and classical YangBaxter c equations, Ann.Inst.Fourier, Grenoble, v.37(1987), p.139-160.

[K2] T.Kohno. Series de Poincare-Koszul associe aux grouppes de tresses pures. Inv.Math. No 82(1985), p.57-75

[Ka1] L.H.Kauffman, An invariant of regular isotopy. Trans.Amer.Math.Soc., vol.318, 2 (1990) 317-371.

[Ka2] L.H.Kauffman, State model and the Jones polynomial. Topology, v.26(1987)p.395-

407.

[Ko1] M.Kontsevich, Vassiliev's knot invariants. Preprint 1992 .

[Ko2] M.Kontsevich, Private communication.

[Ko3] M.Kontsevich, Feynman diagrams and Low-dimensional topology.

Preprint, January 1993. 
[Ko4] M.Kontsevich, Graphs, homotopical algebra and low-dimensional topology.

Pre-preprint, 1992.

[Ko4] M.Kontsevich, in preparation.

[KZ] V.G.Knizhnik and A.B.Zamolodchikov, Current algebra and Wess-Zumino models in two dimensions, Nucl.Phys.B247 (1984)p.83-103.

[Li1] X.S.Lin, Vertex models, quantum group and Vassiliev knot invariants. Preprint, 1991

[Li2] X.S.Lin, Milnor $\mu$-invarianys ar all of finite type. Preprint, 1992.

[Mi1] J.Milnor, Link groups. Ann.of Math.59(1954), p.177-195.

[LM] Le Tu Quoc Thang and J.Murakami. On relation between Kontsevich's integral invariant and invariants coming from quantum R-matrices.

Pre-preprint.January 1993.

[Mi2] J.Milnor, Isotopy of links, Algebraic geometry and Topology (R.H.Fox ed.) Princeton Univ.Press., Princeton, No1, (1957) p.280-306.

[Pi1] S.A.Piunikhin, Weights of Feynmann diagrams, link polynomials and Vassiliev knot invariants. To appear in Journal of Knot Theory and its Ramifications .

[Pi2] S.A.Piunikhin, Vassiliev knot invariants contain more information, than all knot polynomials (To appear in Journal of Knot Theory and its Ramifications) [Pi3] S.A.Piunikhin, Turaev-Viro and Kauffman-Lins Invariants for 3-Manifolds coincide. Journal of Knot Theory and its Ramifications,

vol 1, No2 (1992), p.105-135.

[Pi4] S.A.Piunikhin, State sum model for trivalent knotted graph invariants using the quantum group SL (2).

Journal of Knot Theory and its Ramifications , v.1, No3(1992), p.273-278. [Ro] D.Rolfsen, Knot and Links, (Publish or Perish, 1976).

[Re1] N.Yu.Reshetikhin, Quantized universal enveloping algebras, the YangBaxter equation and invariants of links. LOMI-preprint E-4-87, E-17-87.

[Re2] N.Yu.Reshetikhin, Quasitriangular Hopf algebras and invariants of links. Leningrad, Math J.1 (1990)p.

[RT] N.Yu.Reshetikhin and V.G.Turaev, Ribbon graphs and their invariants derived from quantum groups. Commun.Math.Phys., vol.127, 1(1990) 1-26.

[TK] A.Tsuchiya, Y.Kanie Vertex operators in two dimensional conformal field theory on $\mathbf{P}^{\mathbf{1}}$ and monodromy representations of braid groups.

in Advanced studies in Pure Mathematics. 16. Tokyo, Kinokuniya, 1988, p.297-372.

[Tu] V.G.Turaev, The Yang-Baxter equation and invariants of links.

Invent.Math.v.92(1988)p.527-553.

[Tu] V.G.Turaev, Operator invariants of tangles and R-matrices, Math.UssR, Izvestiya, vol.35., (1990), No2, p.411-444.

[Va1] V.A.Vassiliev, Cohomology of Knot Spaces. Advances in Soviet Mathematics, v.1, AMS(1990), p.23-69 . 
[Va2] V.A.Vassiliev, Complements of discriminants of smooth maps. Topology and applications, Trans.of Math.Mono.98, Amer.Math. Soc., Providence, 1992.

[Wi] E.Witten, Quantum field theory and Jones polynomial.

Commun.Math.Phys., vol.121, No3 (1989) p.351-399. 